\title{
Reaction of Anti-OJ Autoantibodies with Components of the Multi-enzyme Complex of Aminoacyl-tRNA Synthetases in Addition to Isoleucyl-tRNA Synthetase
}

\author{
Ira N. Targoff, Edward P. Trieu, and Frederick W. Miller* \\ Department of Medicine, University of Oklahoma Health Sciences Center, Oklahoma City, Oklahoma; the Arthritis/Immunology \\ Program, Oklahoma Medical Research Foundation, Oklahoma City, Oklahoma 73104; the Veterans Affairs \\ Medical Center, Oklahoma City, Oklahoma 73104; and *the Center for Biologics Evaluation \\ and Research, Food and Drug Administration, Bethesda, Maryland 20892
}

\begin{abstract}
Autoantibodies to five aminoacyl-tRNA synthetases have been reported, and all have been associated with a syndrome of myositis and interstitial lung disease. Four of these synthetases exist free in the cytoplasm, but the fifth, isoleucyl-tRNA synthetase (recognized by anti-OJ autoantibodies), is a component of the multi-enzyme complex containing at least seven synthetases. In an effort to better understand the origins of these antibodies, we examined sera from 11 patients with antiOJ autoantibodies for evidence of reaction with other components of the complex. All sera showed a characteristic pattern of 10 protein bands by immunoprecipitation from HeLa cell extract. 10 of 11 sera significantly inhibited isoleucyl-tRNA synthetase enzyme activity. Serum and IgG from four patients also inhibited leucyl-tRNA synthetase activity, and serum and IgG from two inhibited lysyl-tRNA synthetase. Immunoblotting experiments supported reaction of the two sera with lysyltRNA synthetase, and revealed additional reactivity of three sera with a 160-kD component believed to be glutaminyl-tRNA synthetase. Despite reaction of some sera with additional synthetases, the immunoprecipitated tRNA appeared the same with all sera, and functioned as tRNA ${ }^{\text {ile }}$.

While reaction with more than one synthetase was seen with some anti-OJ sera, all synthetases targeted by anti-OJ sera were components of the complex, rather than unassociated synthetases. These findings suggest that an initial autoantibody response against isoleucyl-tRNA synthetase was followed by extension to involve other components of the synthetase complex. These observations may have implications for understanding the generation of antisynthetase autoantibodies. ( $J$. Clin. Invest. 1993. 91:2556-2564.) Key words: myositis • amino acyl T RNA synthetases $\bullet$ autoimmunity $\bullet$ autoantibodies - interstitial lung disease
\end{abstract}

\section{Introduction}

Autoantibodies to aminoacyl-tRNA synthetases have been closely associated with the autoimmune inflammatory myopa-

This work was presented in part at the 1991 Annual Meeting (November 17-21, 1991) and Central Regional Meeting (March 21, 1991) of the American College of Rheumatology, Boston, MA, and the Second International Workshop on the Molecular and Cell Biology of Autoantibodies and Autoimmunity, San Diego, CA (September 1921, 1991).

Address reprint requests to Dr. Ira N. Targoff, Oklahoma Medical Research Foundation, 825 NE 13th St., Oklahoma City, OK 73104.

Received for publication 1 April 1992 and in revised form 4 December 1992.

The Journal of Clinical Investigation, Inc.

Volume 91, June 1993, 2556-2564 thies, polymyositis (PM) ${ }^{1}$ and dermatomyositis (DM) (1-8), occurring in $\sim 25-30 \%$ of PM/DM patients. They include anti-Jo-1, the most common (9), which reacts with histidyltRNA synthetase (HisRS) (10); anti-PL-7, which reacts with threonyl-tRNA synthetase $(3,4)$; anti-PL-12 with alanyltRNA synthetase and tRNA ${ }^{\text {ala }}(2,6)$; anti-EJ with glycyltRNA synthetase $(5,11)$; and anti-OJ with isoleucyl-tRNA synthetase (IleRS) (5). The autoantibodies to aminoacyltRNA synthetases (antisynthetases) have been associated with a characteristic clinical picture, marked by a high frequency of interstitial lung disease (ILD; $50-75 \%$ of patients $[1,12,13]$ ), arthritis (14), Raynaud's phenomenon, fevers, and other features, that has been referred to as the "antisynthetase syndrome" $(1-4,6,11-14)$. Despite the similarity in the associated clinical picture between patients with different antisynthetases, no individual patient has previously been reported to have autoantibodies to more than one aminoacyl-tRNA synthetase enzyme.

Anti-OJ antibody was originally identified when the two original sera from patients $\mathrm{OJ}$ and $\mathrm{NJ}$ were found to immunoprecipitate identical tRNA and protein bands from HeLa cell extract. Serum and IgG from these patients strongly and specifcally inhibited the enzymatic activity of IleRS, which catalyzes the formation of Ile-tRNA from Ile and tRNA ${ }^{\text {ile }}(5)$. In higher eukaryotic cells, 7-9 of the 20 synthetases (those for glutamine, isoleucine, leucine, methionine, glutamic acid, arginine, lysine, and sometimes aspartic acid and/or proline) are found together in a stable, high molecular weight complex along with three other nonsynthetase proteins $(15,16)$. Almost all of the enzymatic activity of IleRS is found associated with this multienzyme complex. The other four synthetases against which human autoantibodies have been identified are usually found free in the cytoplasm, and they are not found in purified complexes. The 10 protein bands immunoprecipitated by $\mathrm{OJ}$ and $\mathrm{NJ}$ sera were consistent in molecular weight with those of the multi-enzyme complex (5).

Using sera from 11 patients that contained anti-OJ autoantibodies as determined by immunoprecipitation (IP) of the synthetase complex pattern, we addressed the questions of whether all anti-OJ sera react with the same component of the synthetase complex, and whether any react with multiple components. Our findings revealed a complicated picture that may provide important insights into the mechanisms for the development of antisynthetases in particular and other autoantibodies to ribonucleoprotein complexes generally.

1. Abbreviations used in this paper: DM, dermatomyositis; HisRS, histidyl-tRNA synthetase; ILD, interstitial lung disease; IleRS, isoleucyl-tRNA synthetase; IP, immunoprecipitation; LeuRS, leucyltRNA synthetase; LysRS, lysyl-tRNA synthetase; PM, polymyositis. Two-letter codes indicate individual patients. 


\section{Methods}

Sera. Sera from $>1,000$ patients were tested by IP, including sera from patients with proven or suspected myositis from clinics at the University of Oklahoma Health Sciences Center or the National Institute for Arthritis, Musculoskeletal and Skin Diseases; sera referred from other centers; and sera with anticytoplasmic antibodies by indirect immunofluorescence even if there was no suspicion of myositis. Many of the patients had been included in previous studies $(4-6,12,17,18)$. Those showing the anti-OJ pattern were identified. "Original anti-OJ sera" refers to the two sera from patients described in the previous study of anti-OJ ( NJ and OJ) (5). Two sera with anti-OJ (RH and GZ) that had been included in a large study of myositis (12) were included here with the "new" sera since immunological analysis had not previously been described. Two other samples with anti-OJ were excluded because the patient of origin could not be confirmed.

Immunoprecipitation. IPs for protein and nucleic acid analyses were performed as previously described (5). For the former, protein A-Sepharose (1-2 mg when dry) was coated with $10 \mu \mathrm{l}$ of test serum and incubated with extract of $2 \times 10^{5} \mathrm{HeLa}$ cells per sample that had been labeled overnight with $\left[{ }^{35} \mathrm{~S}\right]$ methionine. Immunoprecipitates were analyzed by $10 \%$ SDS-PAGE and developed with autoradiography. For nucleic acids, 3-5 mg of protein A-Sepharose was coated with $20 \mu \mathrm{l}$ of serum and incubated with extract of $6 \times 10^{6}$ unlabeled HeLa cells. Immunoprecipitates were phenol-extracted, analyzed by $7 \mathrm{M}$ urea-10\% PAGE, and developed with silver stain (Bio-Rad Laboratories, Richmond, CA).

Immunoblotting. The antigen source for immunoblotting was either whole HeLa cell extract prepared as for IP (5) or immunoprecipitate of anti-OJ sera from HeLa cell extract. The procedure for immunoblotting using whole extract was as previously described (19) with the following modifications. After 8-9\% SDS-PAGE and transfer to nitrocellulose, strips were blocked with $5 \%$ bovine nonfat milk in Tris-saline blotting buffer. Sera were diluted 1:100 in this milk solution. Goat anti-human $\operatorname{IgG} /$ alkaline phosphatase was used as conjugate and bromo-chloro-indolyl-phosphate/nitroblue tetrazolium as substrate (Kierkegaard \& Perry Laboratories, Gaithersburg, MD).

Immunoblotting of immunoprecipitates was performed to determine whether immunoblot bands represented complex components. IP was performed using unlabeled cells prepared as above for nucleic acid IP, but the quantities of serum, cells, and Sepharose were increased fourfold per sample. Immunoprecipitates were electrophoresed and transferred to nitrocellulose, strips were developed as for immunoblot, but were blocked overnight, and a protein $\mathrm{A}$ /alkaline phosphatase conjugate was used. To determine which IP bands were recognized in immunoblot, ${ }^{35} \mathrm{~S}$-labeled extract was used for IP, and an autoradiograph of the nitrocellulose was taken after transfer. The portion of the nitrocellulose above the $60-\mathrm{kD}$ molecular mass level was developed as above for immunoblotting. The nitrocellulose strips were positioned as in the original sheet, and then overlaid with the autoradiograph.

Antibody was affinity-purified from immunoblots by identifying and excising the band of interest, blocking, incubating with sera diluted $1: 100$, and washing as for immunoblotting. Specific antibody was eluted by incubation for $2 \mathrm{~min}$ at $4^{\circ} \mathrm{C}$ in $0.2 \mathrm{M}$ glycine buffer, $\mathrm{pH} 2.8$, with $0.15 \mathrm{M} \mathrm{NaCl}$ and $0.05 \%$ Tween 20 , followed by immediate neutralization with $1 \mathrm{M}$ Tris. The entire $0.5 \mathrm{ml}$ eluate containing affinity-purified antibody was then used for immunoprecipitation or was mixed with $0.125 \mathrm{ml}$ of $20 \%$ milk and used for immunoblotting. In some cases, $3 \mathrm{M} \mathrm{Na}$ thiocyanate was used for elution and removed with dialysis.

Aminoacylation inhibition. Each aminoacyl-tRNA synthetase catalyzes an aminoacylation reaction ( tRNA charging) in which its particular amino acid is covalently linked to the cognate tRNA. The ability of sera to inhibit the activity of each synthetase was tested individually as described previously $(5,19)$. The tRNA charging reaction for each amino acid was set up using amino acid labeled with ${ }^{3} \mathrm{H},{ }^{35} \mathrm{~S}$, or ${ }^{14} \mathrm{C}$; whole calf liver tRNA (Boehringer Mannheim Corp., Indianapolis, IN); and, as enzyme source, HeLa cell extract or, in early experiments, calf liver extract. The enzyme source was preincubated with patient or control serum, usually diluted $1: 10$. Activity was tested at 10 and 20 $\mathrm{min}$ as the radioactivity incorporated into the trichloroacetic acid precipitate. The percentage of inhibition $=[($ activity with normal sera - activity with patient sera $) \div$ (activity with normal sera) $] \times 100$. As a result of nonspecific inhibition by some sera, and as determined by previous studies, inhibition by a serum was considered significant only if $>50 \%$ of activity was inhibited as compared with the average results with at least two normal sera. For confirmation of significant inhibition, purified IgG was obtained by DEAE chromatography as described previously $(5,19)$.

To determine the identity of the immunoprecipitated tRNA, IP reactions using $O J$ serum were performed as described above until sufficient tRNA was obtained. The tRNA was isolated by phenol extraction and ethanol precipitation, and used in a standard aminoacylation reaction. The immunoprecipitated tRNA was tested for the ability to accept each of the amino acids whose synthetase is consistently found in the multi-enzyme complex except arginine, which was unavailable in labeled form at the time of this portion of the study. Results were standardized on a molar basis (picomoles of amino acid incorporated into the precipitate) and standardized between experiments by comparison to charging with isoleucine.

\section{Results}

\section{Autoantibody identification}

IP with sera from nine new patients showed a protein pattern that was identical to that of sera from the original two anti-OJ patients (Fig. 1). Since the pattern was composed of 10 consistent bands (Fig. 1, $a-j$ ), it could be specifically identified from protein IP gels alone. With each of the sera, the three protein bands of highest molecular weight were much more intense than the seven other bands of the OJ complex. At least five sera immunoprecipitated one or more additional proteins that were not shared and did not appear to be part of the complex, including U1RNP proteins with serum JM, Ro/SSA protein with serum RB, both Ro/SSA and La/SSB proteins with serum RH, and unidentified proteins with sera $\mathrm{NJ}$ and $\mathrm{WH}$.

The nine new sera that immunoprecipitated the protein pattern all immunoprecipitated tRNA bands from HeLa cells that appeared identical to those of the original two sera, consisting of one faster migrating, more intense band and one weaker band (Fig. 2). A third weak, fast migrating band that may have been a degradation product was seen in some experiments. The pattern could be distinguished from the tRNA bands seen with other antisynthetases (Fig. 2). The combination of protein and nucleic acid bands by IP confirmed the anti-OJ antibody specificity. The Ro/SSA RNAs were seen with sera RH and RB, the La/SSB RNAs with serum RH, the U1 RNA and an unidentified 7S RNA with JM, and an unidentified 4.5-S RNA similar in migration to the Mas RNA with JX (12).

\section{Determination of the components reactive with autoantibodies}

IP would not necessarily distinguish between sera reacting with different components of the complex. Aminoacylation inhibition and immunoblot were used to determine the antigenic components.

\section{AMINOACYLATION INHIBITION}

At the screening dilution of $1: 10$, the original anti-OJ sera again showed consistent significant inhibition of IleRS activity by 98-100\% (Table I) (5), while control anti-Jo-1 serum, which 


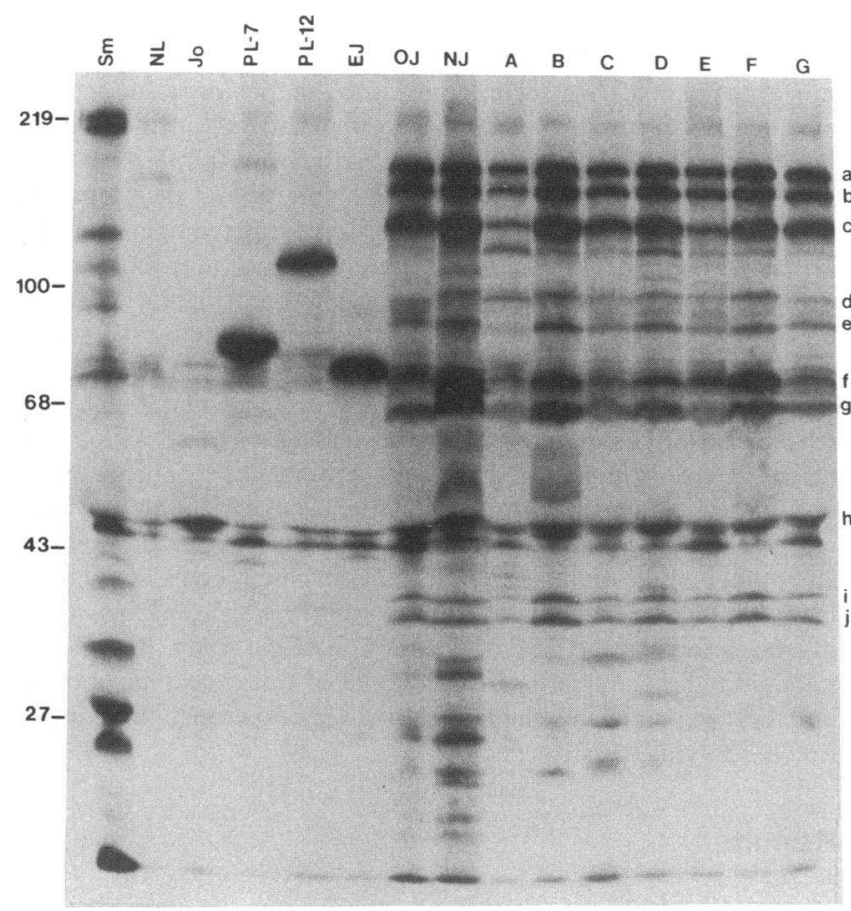

Figure 1. Immunoprecipitation from $\left[{ }^{35} \mathrm{~S}\right]$ methionine-labeled HeLa cell extracts with anti-OJ sera. 10\% SDS-PAGE of immunoprecipitates using seven new sera $(A-G)$ and the original anti-OJ sera $\mathrm{NJ}$ and OJ. $N L$, normal. Standard sera for the indicated autoantibodies were used for lanes marked $S m, J o, P L-7, P L-12$, and $E J$. The positions of molecular weight markers are indicated in kilodaltons on the left. Lanes $A-G$ show results with the following sera: $A, \mathrm{AC} ; B, \mathrm{RH} ; C$, $\mathrm{JM} ; D, \mathrm{JX} ; E$, JS; $F, \mathrm{JH} ; G, \mathrm{WH}$. The same characteristic pattern of 10 protein bands $(a-j)$ is seen with each of the nine anti-OJ sera shown. The pattern with GZ and RB, not shown, was similar. Artifacts are seen at 45, 50, and $75 \mathrm{kD}$. Bands $a-h$ are believed to represent synthetases for the following amino acids (Thommes et al. [20]): $a$, glutamine (or glutamic acid with or without proline (Mirande [15]); $b$, isoleucine; $c$, leucine; $d$, methionine; $e$, glutamic acid (or glutamine); $f$, lysine; $g$, arginine; $h$, aspartic acid. Bands $i$ and $j$ are nonsynthetase components.

strongly inhibited HisRS, did not inhibit IleRS. For six of nine new patients, initial serum samples consistently showed very strong inhibition of IleRS $(>80 \%)$. Serum of two others ( $\mathrm{JH}$ and WH) also inhibited IleRS significantly (50-80\%). Serum of the ninth patient, JS, which was the weakest of the initial samples by IP, showed no IleRS inhibitory activity, even when tested undiluted. Samples drawn at a later date from four patients whose initial serum showed very strong inhibition were tested. Two showed a decrease in activity, AC to $32-64 \%$ (shown in Table I) and GZ to 64-88\%. AC also showed clearly decreased IP activity.

To confirm that this inhibition represented antibody activity, purified IgG was tested. IgG from six of nine new sera significantly inhibited (>50\%) IleRS activity at $1 \mathrm{mg} / \mathrm{ml}$, while two ( $\mathrm{JH}$ and follow-up $\mathrm{AC}$ ) required $3-6 \mathrm{mg} / \mathrm{ml}$ for significant inhibition. IgG from the ninth serum, noninhibitory JS serum, showed partial inhibition only at $6 \mathrm{mg} / \mathrm{ml}$.

Aminoacylation inhibition assays were performed for all 20 amino acids on serum from each of the 11 anti-OJ patients, including the two original patients whose serum had been tested previously (5). Results for those amino acids for which

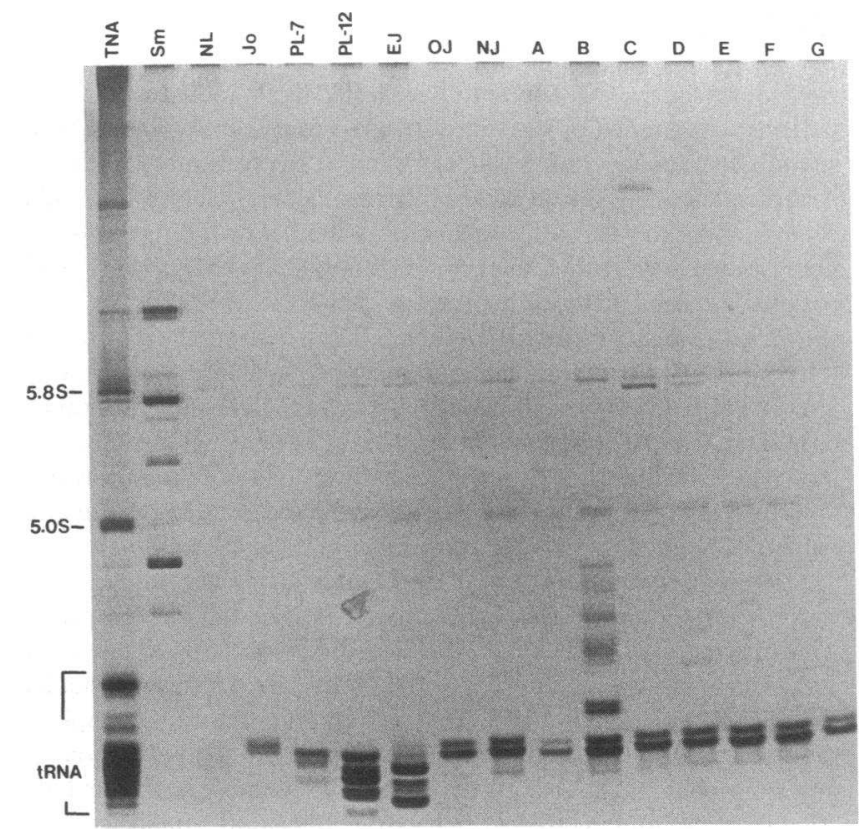

Figure 2. Immunoprecipitation from HeLa cells developed with silver stain. $7 \mathrm{M}$ urea, 10\% PAGE of nucleic acid obtained by phenol extraction of immunoprecipitates using seven new sera (lanes $A-G$ as in Fig. 1) and original anti-OJ sera NJ and OJ. Standard sera were used in lanes $S m, J o, P L-7, P L-12$, and $E J$. TNA, total nucleic acid. The positions of 5.0S and 5.8S ribosomal RNAs and TRNA are indicated. All nine sera immunoprecipitated tRNA in a characteristic pattern. RB and GZ (not shown) were identical. Other bands immunoprecipitated include Ro and La RNAs in lane $B$, U1 RNA in lane $C$, and unidentified RNAs in lanes $C$ and $D$.

one or more sera showed consistent significant inhibition are shown in Table I. Initial serum samples from five of the new patients significantly inhibited leucyl-tRNA synthetase (LeuRS) activity, including two (JM, JH) that inhibited $>80 \%$. Significant inhibition could be confirmed with purified IgG from four of the five, including inhibition of $93 \%$ by JMIgG. Inhibition of LeuRS was less than inhibition of IleRS for each individual serum except $\mathrm{JH}$. With patients $\mathrm{GZ}$ and $\mathrm{AC}$, initial serum samples significantly inhibited LeuRS but subsequent samples did not.

Two sera, JH and RB, strongly inhibited lysyl-tRNA synthetase (LysRS) $(>80 \%)$. This was confirmed with $\mathrm{IgG}$ purified from each serum, which inhibited 78-96 and 100\%, respectively. Both sera inhibited LysRS more than IleRS. JH-IgG significantly inhibited LysRS and LeuRS but not IleRS at 1 $\mathrm{mg} / \mathrm{ml}$. Serum RB also strongly inhibited arginyl-tRNA synthetase (92\%), but inhibition with RB-IgG was consistently below the significance level $(41 \%)$.

\section{IMMUNOBLOTTING}

Several specific bands were identified which were stained strongly and reproducibly by at least one serum, but no band was shared by the majority of sera. Several bands were initially considered as possible candidates for components of the synthetase complex (Table II), including a $160-\mathrm{kD}$ band, stained by sera of three patients $(\mathrm{JH}, \mathrm{AC}$, and $\mathrm{NJ}) ; 150$ - and $140-\mathrm{kD}$ bands stained by one each (JS and GZ); and a 48-kD band stained by four (OJ, NJ, JS, and GZ) (Fig. 3). Other unidentified bands were stained by single sera, such as a $100-\mathrm{kD}$ band 
Table I. Percent Inhibition of Aminoacylation for Each Amino Acid by Anti-OJ Sera

\begin{tabular}{|c|c|c|c|c|c|c|c|c|c|c|c|c|}
\hline & OJ & NJ & $\mathrm{AC}^{*}$ & JS & RH & $G Z^{\ddagger}$ & JH & JX & $\mathrm{JM}$ & $\mathbf{W H}^{\ddagger}$ & $\mathbf{R B}^{\ddagger}$ & Jo-1 \\
\hline Ile & 100 & 99 & $32 *$ & 1 & 90 & 100 & 83 & 99 & 100 & 59 & 80 & 0 \\
\hline Leu & 7 & 87 & $12^{*}$ & 11 & 54 & 76 & 74 & 81 & 95 & 0 & 5 & 0 \\
\hline Lys & 33 & 39 & 0 & 0 & 0 & 0 & 81 & 0 & 4 & 0 & 100 & 0 \\
\hline Ile-IgG & 95 & $\mathrm{ND}^{\S}$ & 11 & 4 & 80 & 99 & 0 & 58 & 99 & 53 & 76 & 0 \\
\hline Leu-IgG & 0 & ND & 0 & 28 & 30 & 76 & 82 & 68 & 93 & 13 & 5 & 10 \\
\hline Lys-IgG" & 0 & ND & 8 & 15 & 0 & 19 & 78 & 0 & 0 & 0 & 100 & 33 \\
\hline Gln" & 7 & 33 & 13 & 12 & 0 & $12^{1}$ & 26 & 13 & 0 & 4 & 14 & $15^{\ddagger}$ \\
\hline His" & 5 & 27 & 6 & 3 & 0 & 3 & 0 & 0 & 5 & 0 & 0 & 94 \\
\hline Arg" & 0 & 0 & 0 & 0 & 15 & 23 & 35 & 0 & 6 & 8 & $92^{* *}$ & 0 \\
\hline
\end{tabular}

Patient designations are indicated across the top, and amino acids tested are shown at the left. Values are percentage of inhibition of aminoacylation as compared with the average of two to four normals included with each test, calculated as: [(counts/minute with average normal counts/minute with test $) \div$ (counts/minute with average normal) $] \times 100$. All readings for a given amino acid were derived from the same experiment except where indicated. Values $<0$ are shown as 0 , and $>100$ as 100 . ND, not done. All readings are shown for 1:10 dilution of serum or $1 \mathrm{mg} / \mathrm{ml}$ of isolated IgG before addition to the reaction mixture. Jo- $1=$ anti-Jo-1 standard serum. Sera OJ and NJ had also been previously tested (5). No anti-OJ serum showed consistent significant inhibition of synthetases for the other 14 amino acids that are not shown.

* All results shown for this patient are for follow-up serum. The initial sample, which had much stronger anti-OJ activity by IP, was depleted, but had inhibited IleRS $81 \%$ and LeuRS $60-81 \%$ in previous studies.

‡ All tests for RB, all for WH except Gln, and all for GZ-IgG, were performed separately; 10-min readings are shown.

${ }^{\S}$ Serum was depleted; tested previously (5).

"10-min readings (others are 20 -min readings).

' Follow-up serum, which had reduced anti-IleRS activity compared with original serum, was used for this test because initial serum was depleted.

** RB-IgG showed $41 \%$ inhibition (not significant).

by $\mathrm{JX}$. In addition, staining related to coexisting antibodies to Ro/SSA, La/SSB, and U1RNP was seen, as expected from the IP results.

The molecular masses suggested that the 160-, 150-, and $140-\mathrm{kD}$ bands might have been bands $a, b$, and $c$, respectively, of the complex. The 48-kD band was closest in size to band $h$, but migrated faster. Two approaches were taken to determine whether these bands were components of the synthetase complex: ( $a$ ) affinity purification of antibodies to each of the bands, and $(b)$ immunoblotting of the immunoprecipitated synthetase complex.

Affinity-purified autoantibodies. Affinity-purified autoantibodies to the proteins of $160 \mathrm{kD}$ (from NJ and AC), $150 \mathrm{kD}$ (from JS), $140 \mathrm{kD}$ (from GZ), and $48 \mathrm{kD}$ (from OJ, JS, and GZ) were obtained by elution from immunoblots and tested in immunoprecipitation (Fig. 4). In each case, the presence of active antibody without other significant bands was confirmed by restaining in immunoblot (not shown). Anti-OJ proteins $a$, $b$, and $c$ were visible in immunoprecipitates of affinity-purified anti-160-kD antibodies. However, the bands were very weak despite intense staining by these antibodies in immunoblot. Affinity-purified anti-150-kD from JS serum also immunoprecipitated anti-OJ-associated proteins, and the protein bands were much more intense than with anti-160 kD. The $a, b$, and $c$ proteins were also seen with affinity-purified anti-48 $\mathrm{kD}$ from JS serum, but they were not as strong as with anti-150 kD of JS serum, and they were not seen with anti-48 $\mathrm{kD}$ of OJ, GZ, or $\mathrm{NJ}$ sera. The reason for this difference in results with anti-48 $\mathrm{kD}$ from different sera was unclear.

Affinity-purified anti-140-kD antibodies did not show IP of anti-OJ-associated proteins. None of the affinity-purified antibodies showed detectable tRNA on IP. These data suggested that the 160 - and $150-\mathrm{kD}$ immunoblot bands were components of the synthetase complex and that the $140-\mathrm{kD}$ band was not. This method could not demonstrate definitively which synthetase complex components corresponded to the 160 - and 150-kD bands.

\section{Table II. Major Bands Recognized in Immunoblotting} by Anti-OJ Sera

\begin{tabular}{lllllll}
\hline $\begin{array}{l}\text { Western blot } \\
\text { band }\end{array}$ & $160 \mathrm{kD}$ & $150 \mathrm{kD}$ & $140 \mathrm{kD}$ & $75 \mathrm{kD}^{*}$ & $48 \mathrm{kD}$ & Other \\
\hline IP band & $a$ & $b$ & None & $f$ & None & \\
Enzyme & GlnRS & IleRS & & LysRS & & \\
OJ & - & - & - & - & + & $43 \mathrm{kD}$ \\
NJ & + & - & - & - & + & - \\
AC & + & $\pm^{\S}$ & - & - & - & Series \\
JS & - & + & - & - & + & - \\
RH & - & - & - & - & - & Ro/La \\
JX & - & - & - & - & - & $100 \mathrm{kD}$ \\
JH & + & - & - & + & - & $60 \mathrm{kD}$ \\
GZ & - & - & + & - & + & - \\
WH & - & - & - & - & - & - \\
RB & - & - & - & + & - & $70 \mathrm{kD}$ \\
JM & - & - & - & - & - & RNP \\
\hline
\end{tabular}

Sera that react with each of the major immunoblot bands are indicated $(+$, reactive, - , negative, \pm , equivocal). Western blot bands are indicated by molecular mass in kilodaltons. Other = additional bands stained by the serum. IP bands (immunoprecipitated complex components) are as in Fig. 1. None = no component of the immunoprecipitated complex corresponds to that Western blot band. Enzymes indicated are believed to correspond to the immunoprecipitated bands as in Fig. 1. Sera are designated to the left by letters as in the text. * Detected only in blots of immunoprecipitates. ${ }^{\ddagger}$ Some disagreement as to identity; see Fig. 1 and text. ${ }^{\S}$ Serum AC stains a strong band of $160 \mathrm{kD}$ and a series of weaker bands, one of which is similar in size to the $150-\mathrm{kD}$ band. 


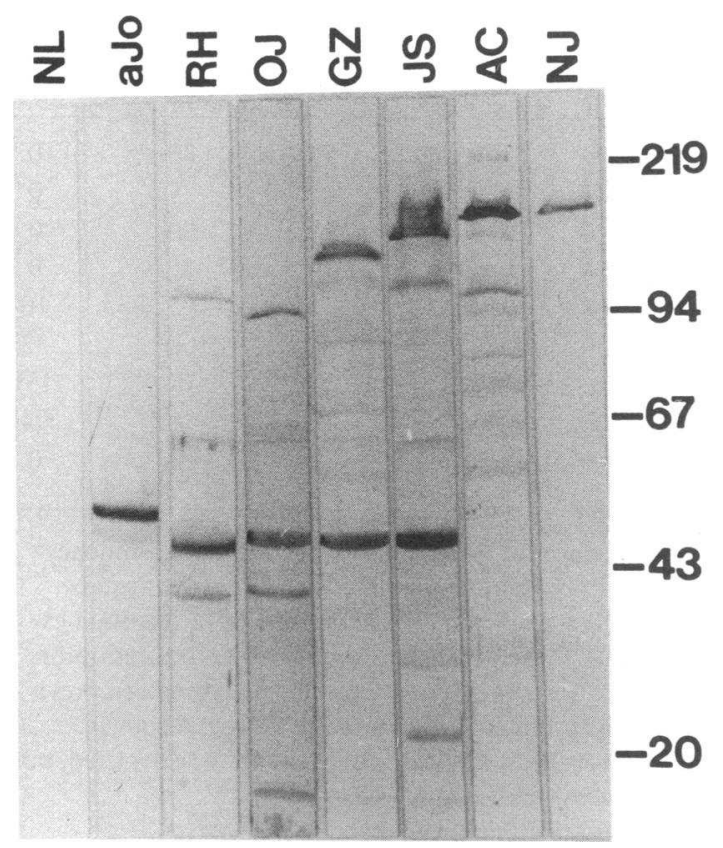

Figure 3. Immunoblot against whole HeLa cell extract. 8\% SDSPAGE was performed, and proteins were transferred to nitrocellulose and processed as in Methods. Sera used for immunostaining were: $N L$, normal; $a J o$, anti-Jo-1 standard serum; $O J$ and $N J$, original antiOJ sera; and $R H, G Z, J S$, and $A C$, new anti-OJ sera. The positions of molecular mass markers in kilodaltons are shown at the right. Significant bands include the $48-\mathrm{kD}$ band seen with $\mathrm{OJ}, \mathrm{GZ}$, and JS; the $140-\mathrm{kD}$ band with GZ; the $150-\mathrm{kD}$ band with JS; the $160-\mathrm{kD}$ band with $\mathrm{AC}$ and $\mathrm{NJ}$; the $50-\mathrm{kD}$ band of Jo-1; and the $45-\mathrm{kD} \mathrm{La} / \mathrm{SSB}$ band in lane $R H$. The NJ sample originally had anti-48-kD activity which was lost over time and is barely visible.

Immunoblotting of immunoprecipitated complexes. When immunoprecipitates obtained using serum $\mathrm{RH}$ were used as antigen source for immunoblotting, the $160-\mathrm{kD}$ band was detected with JH and AC sera (latter not shown), and the 150-kD band was identified by JS serum (Fig. 5). The 140- and 48-kD bands were not detected by GZ or JS sera. In contrast, when immunoprecipitates were obtained using serum GZ, which reacts with the 140- and 48-kD bands, the $140-\mathrm{kD}$ band was present, as well as the $160-$ and $150-\mathrm{kD}$ bands. The $48-\mathrm{kD}$ band was not detected. The bands were not present in anti-Jo-1 immunoprecipitates, and normal serum did not recognize any bands in any immunoprecipitates. These findings confirmed that the 160- and 150-kD bands were components of the immunoprecipitated OJ antigen complex, and that the 140-kD band was not a component of the complex. Presumably, it was only recognized by a coexisting antibody in GZ serum. The $48-\mathrm{kD}$ band was not identified in the complex by this technique, suggesting that it was not part of the complex, although proximity to the immunoglobulin band may have interfered with detection. In addition to these bands which had been recognized in immunoblots of whole extract, JH and RB sera consistently and strongly stained a 75-kD band in immunoprecipitates. Serum RB also appeared to stain a $70-\mathrm{kD}$ band more weakly (not shown). The reason these bands were visible only with immunoprecipitates may have been because of the larger amount of specific protein present in immunoprecipitates as compared with amounts in whole extract.

By comparison with protein-stained immunoprecipitates, the $160-\mathrm{kD}$ band corresponded in size to band $a$ and the 150$\mathrm{kD}$ band to band $b$. The identity of band $a$ in the human synthetase complex is not settled, but glutaminyl-tRNA synthetase $(20,21)$ or a multifunctional synthetase showing both glutamyl-tRNA synthetase and prolyl-tRNA synthetase activity (22) have been proposed. Protein band $b$ appears to be IleRS, as determined by molecular mass ( $140-150 \mathrm{kD}$ for IleRS) and position in the complex $(15,20)$. The $140-\mathrm{kD}$ protein band was of slightly higher molecular weight than band $c$, consistent with the other evidence that it was not part of the synthetase complex. The $75-\mathrm{kD}$ band of sera $\mathrm{JH}$ and $\mathrm{RB}$ corresponded to band $f$, which appears to be LysRS, consistent with the aminoacylation inhibition data. Of note was that band $f$ was more intense in ${ }^{35} \mathrm{~S}$-immunoprecipitation with $\mathrm{JH}$ and $\mathrm{RB}$ sera than with other sera (see Fig. 1 for JH; RB not shown). The 70-kD band of RB corresponded to band $q$, arginyl-tRNA synthetase. The immunoglobulin heavy chain prevented assessment of the 48-kD band by protein stain. To confirm this identification of
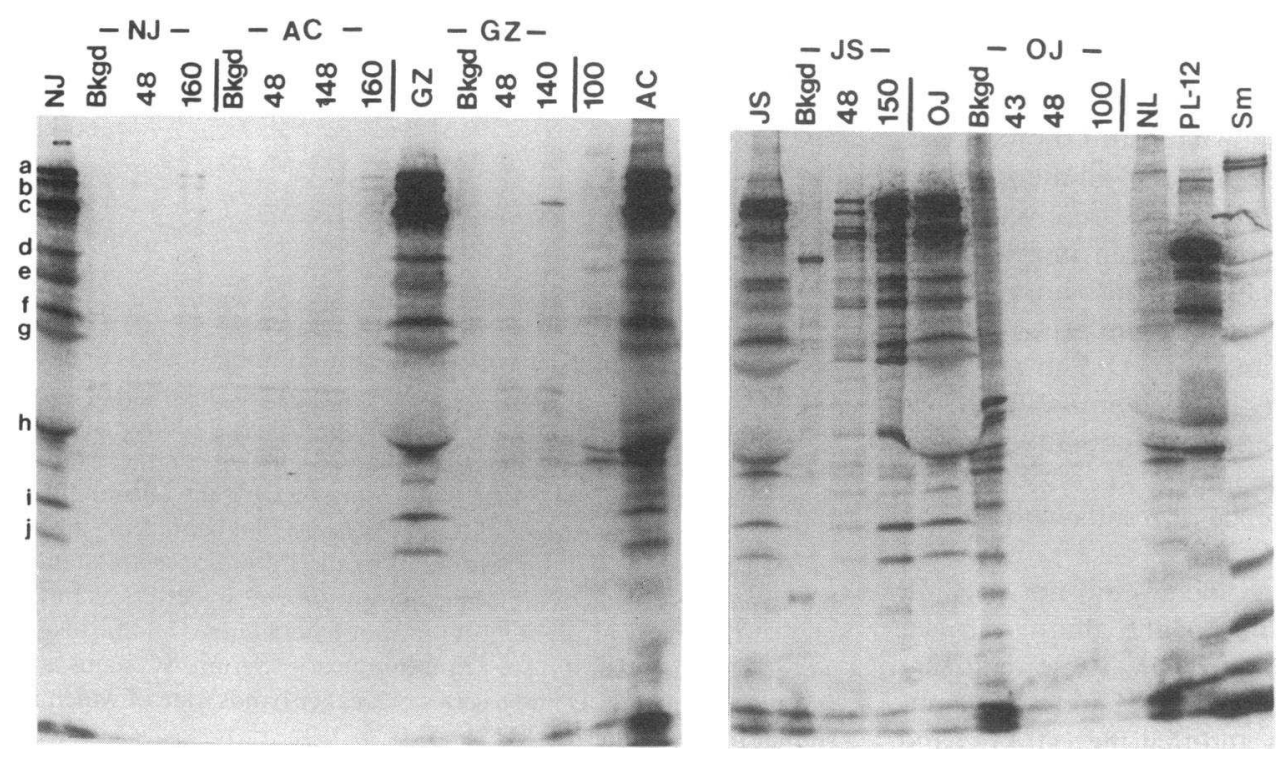

Figure 4. Immunoprecipitation using affinity-purified antibodies. Immunoprecipitation from HeLa cell extract as in Fig. 1 using either whole sera (lanes marked with two letters) or affinity-purified antibodies (lanes marked with numbers, which refer to the molecular mass in kilodaltons of the immunoblot band used). Sera used for affinity purification are indicated horizontally at the top. $B k g d$, background (affinity purification performed on a negative portion of the immunoblot). $N L$, normal. $P L-12$ and $S m$, standard sera. Letters $a-j$ denote the bands of the synthetase complex as in Fig. 1. Bands $a, b$, and $c$ seen with whole sera are faintly visible with affinity-purified antibodies $\mathrm{NJ}$ 160 and AC- 160 , but are much stronger for JS-150 and JS-48. 


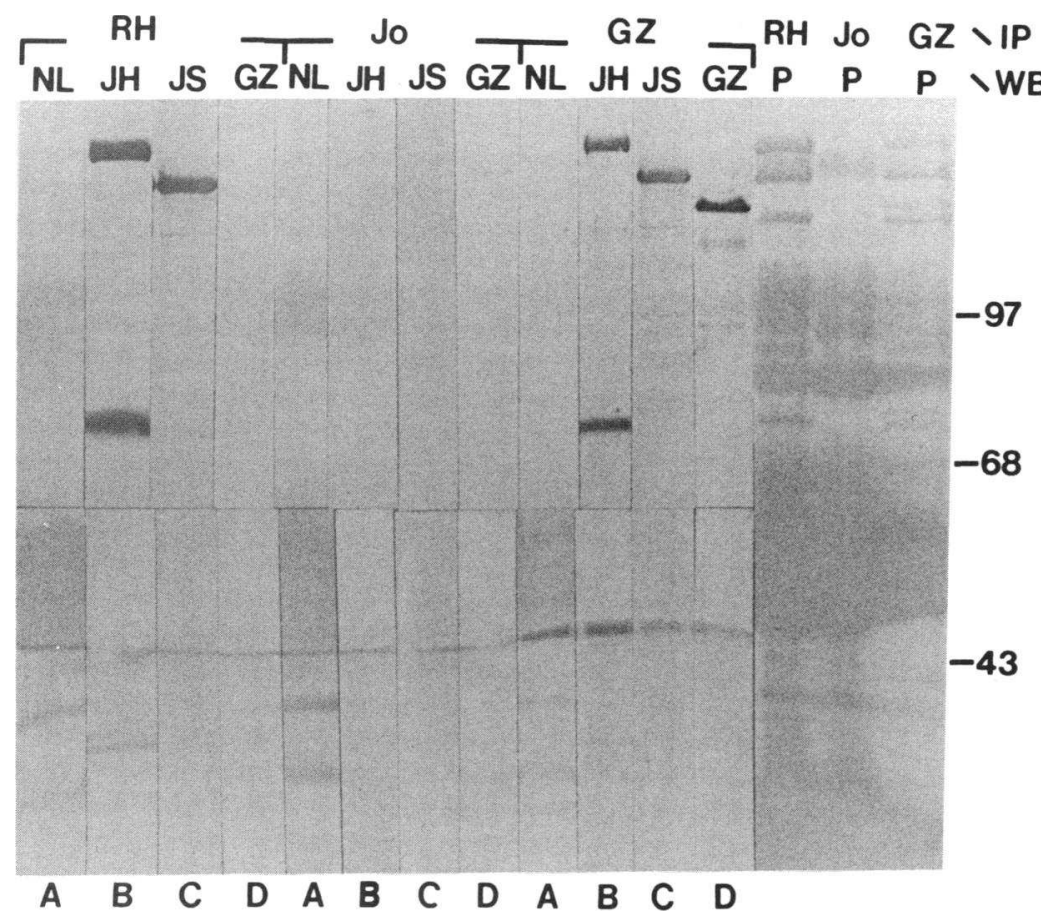

Figure 5. Immunoblot of immunoprecipitates. Immunoprecipitation from HeLa cell extract was performed using anti-OJ sera RH or GZ, or anti-Jo-1 standard serum $(\mathrm{Jo})$, indicated by upper labels $(\backslash I P)$. The immunoprecipitates were analyzed in $8 \%$ SDSPAGE and transferred to nitrocellulose. Immunoblots were developed with protein A-alkaline phosphatase conjugate after incubation with normal serum $(N L)$ or anti-OJ sera $\mathrm{JH}, \mathrm{JS}$, or $\mathrm{GZ}$ as indicated by lower level labels $(\backslash W B)$ or the letters $A-D$, respectively. $P$, fast-green protein stain. The positions of molecular mass markers are indicated in kilodaltons on the right. Against whole extract, these sera showed: $N L$, negative; $J H, 160 \mathrm{kD} ; J S, 150 \mathrm{kD}+48 \mathrm{kD} ; G Z, 140 \mathrm{kD}$ $+48 \mathrm{kD}$; and $R H, \mathrm{La} / \mathrm{SSB}$ (45 kD). The 160-, 150-, and $75-\mathrm{kD}$ bands are seen in RH and GZ but not anti-Jo-1 immunoprecipitates. The $140-\mathrm{kD}$ band is seen only in GZ immunoprecipitates. The $48-\mathrm{kD}$ band is not seen. By comparison to protein stain, the $160-\mathrm{kD}$ band corresponded to band $a$, the $150-\mathrm{kD}$ band to band $b$, and the 75-kD band to band $f$. The 140-kD band did not correspond to band $c$. bands, RH immunoprecipitates from ${ }^{35}$ S-labeled HeLa cell extract were electrophoresed and transferred to nitrocellulose, which was developed by both autoradiography and immunoblotting (see Methods). The 160-, 150-, and 75-kD bands exactly corresponded to bands $a, b$, and $f$, respectively. Immunoblot findings are summarized in Table II.

\section{Identification of immunoprecipitated $t R N A$}

Immunoprecipitated tRNA prepared using standard OJ serum was tested for acceptor activity for each of the amino acids except arginine whose synthetase is consistently associated with the multi-enzyme complex (see Methods). Aspartic acid, which has been found in the complex in some studies, was also tested. Fivefold more isoleucine on a molar basis was accepted by immunoprecipitated tRNA than the next closest amino acid, glutamic acid (Fig. 6).

\section{Clinical features}

Clinical information was available for eight new patients and patient OJ (Table III). Six satisfied criteria for probable or definite PM/DM (23). Two of the others had an elevated creatine kinase level with minimal (OJ) or no (WH) weakness, and did not undergo electromyography or muscle biopsy. One (AC) had no sign of myositis. ILD with minimal or no myositis has been seen with other antisynthetases (6). Eight of nine patients had clinically significant ILD, leading to death in two (OJ and $\mathrm{JH}$ ). The ninth patient (JS) was not known to have ILD, but records were not available for review. All patients with anti-OJ had either PM/DM or ILD or both. Arthritis was common (six of seven), including two patients ( $\mathrm{AC}$ and $\mathrm{JH}$ ) that had a long history of arthritis before the onset of other problems. Raynaud's phenomenon was found in only one of seven. One patient (JS) had DM with malignancy (pancreatic cancer), and one (AC) had Sjögren's syndrome and membranous glomerulonephritis. Overall, anti-OJ was commonly as- sociated with the antisynthetase syndrome observed with other antisynthetases.

\section{Discussion}

These studies showed that IleRS was the major antigen for most, and possibly all, sera with autoantibodies that immunoprecipitated the multi-enzyme complex of aminoacyl-tRNA synthetases. There are at least 10 components of the in vivo complex (15), and antibodies to any component of the synthetase complex would theoretically immunoprecipitate the same complex and thus appear as anti-OJ in screening tests. Therefore, this strong tendency to select IleRS as the preferred antigen of the complex is noteworthy.

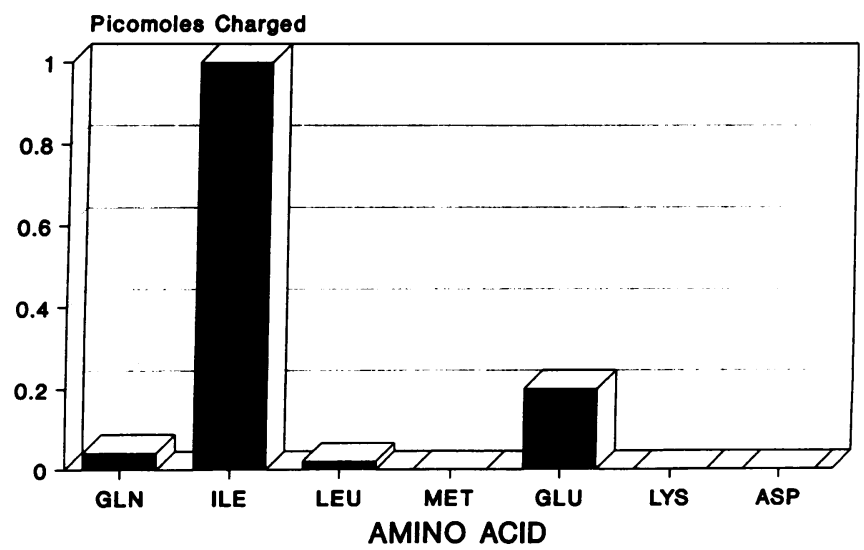

Figure 6. Charging of the immunoprecipitated tRNA associated with $\mathrm{OJ}$ antigen. Immunoprecipitate prepared using $\mathrm{OJ}$ serum and HeLa cell extract was phenol extracted and used as the tRNA source in the aminoacylation reaction, using each of the indicated radiolabeled amino acids. Charging was adjusted on a molar basis. 


\begin{tabular}{|c|c|c|c|c|c|c|c|c|c|c|}
\hline & OJ & AC & RH & GZ & JS & $J X$ & JH & RB & WH & Total $^{*}$ \\
\hline Age (yr) & 53 & $50^{\ddagger}$ & 47 & 60 & Ad & 35 & $75^{\ddagger}$ & 57 & 36 & 52 (average) \\
\hline Sex & $F$ & $\mathrm{~F}$ & $\mathbf{F}$ & $\mathrm{F}$ & $\mathbf{M}$ & $\mathbf{M}$ & $F$ & $\mathbf{M}$ & $\mathbf{M}$ & $5 \mathrm{~F} / 4 \mathrm{M}$ \\
\hline Race & B & B & W & A & $\mathbf{W}$ & B & W & W & W & $3 \mathrm{~B} / 5 \mathrm{~W} / 1 \mathrm{~A}$ \\
\hline Myositis & $\pm^{8}$ & - & + & + & + & + & + & + & $\pm^{\S}$ & $8 / 9^{\prime \prime}$ \\
\hline DM rash & - & - & + & + & + & - & - & - & - & $3 / 9$ \\
\hline ILD & + & + & + & + & -1 & + & + & + & + & $8 / 9$ \\
\hline Arthritis & - & + & + & NA & NA & + & + & + & + & $6 / 7$ \\
\hline Raynaud's & - & - & - & NA & NA & - & + & - & - & $1 / 7$ \\
\hline
\end{tabular}

The presence $(+)$ or absence $(-)$ of each of the key manifestations of the antisynthetase syndrome (listed at the left) is indicated for each of nine anti-OJ-positive patients with clinical data available (indicated across the top). NA, data not available; F, female; M, male; Ad, adult (exact age not available); B, black; W, white; A, Asian.

* Except as indicated, No. positive/No. with information available.

${ }^{\ddagger} \mathrm{AC}$ and $\mathrm{JH}$ had long prior history of arthritis (20 yr for $\left.\mathrm{AC}\right)$.

${ }^{\S} \mathrm{OJ}$ and WH had signs of myositis (both with elevated CK, OJ with minimal weakness) but electromyography or biopsy was not performed.

" Eight of nine had signs of myositis and six of nine had confirmed myositis.

'No known ILD, but records were not available for review.

Of particular significance, however, was the finding that some sera reacted with more than one component of the complex. This demonstrates additional antisynthetase activity in myositis beyond the five synthetases known to be autoantigens, and provides the only instance in which individual patient sera reacted with more than one synthetase. In addition to autoantibodies against IleRS, anti-OJ sera with autoantibodies to LysRS and the $160-\mathrm{kD}$ synthetase protein were demonstrated, and evidence of antibodies to LeuRS and possibly to arginyltRNA synthetase was seen. Serum JH appeared to have activity against four different synthetases, while RB and AC may have reacted with three.

The most convincing evidence was for reaction of $\mathrm{JH}$ and RB sera with LysRS, because aminoacylation inhibition data were confirmed by immunoblotting data. RB also showed both inhibition and immunoblotting of arginyl-tRNA synthetase, but inhibition could not be confirmed at the $>50 \%$ level with purified IgG. The very strong reaction with LysRS raises the possibility that LysRS, rather than IleRS, was the primary antigen for these sera. Against this, however, was the fact that the tRNA pattern was unchanged.

Three sera reacted in immunoblot with the highest molecular weight component of the synthetase complex $(160 \mathrm{kD})$. Studies of the cDNA sequence coding for the largest component ( reported as $170 \mathrm{kD}$ ) noted homology with bacterial glutaminyl-tRNA synthetase and assigned the identity of glutaminyl-tRNA synthetase to the protein on that basis $(20,21)$. However, previous studies of the complex in other mammals identified the highest molecular weight component as glutamyl-tRNA synthetase (15). Later, prolyl-tRNA synthetase activity was also associated with this band (15). A recent study in which cDNAs coding for portions of the homologous protein $(190 \mathrm{kD})$ of Drosophila were expressed in Escherichia coli showed that the amino-terminal portion of the protein had glutamyl-tRNA synthetase activity, while the carboxy-terminal portion had prolyl-tRNA synthetase activity (22). Anti$160 \mathrm{kD}$ in anti-OJ sera apparently did not inhibit $160-\mathrm{kD}$ enzymatic activity, since the three sera did not significantly inhibit glutaminyl-tRNA synthetase, glutamyl-tRNA synthetase, or
prolyl-tRNA synthetase. Anti-160 kD differed in this way from other autoantibodies to synthetases, but resembled antisera of animals immunized with synthetases, which often do not inhibit the synthetase's activity $(24,25)$.

Evidence for the presence of anti-LeuRS was obtained from inhibition studies showing that sera and IgG from certain patients consistently inhibited LeuRS activity to a significant degree. Since no direct binding could be demonstrated to this synthetase, the evidence is not as conclusive as for LysRS and $160 \mathrm{kD}$. Steric inhibition by binding of antibody to another synthetase, such as IleRS, cannot be entirely excluded. However, serum OJ strongly inhibited IleRS without any inhibition of LeuRS, IgG of serum JH showed strong inhibition of LeuRS but very weak inhibition of IleRS, and no other synthetase was sterically inhibited.

An additional finding of this study was the presence of autoantibodies to a $48-\mathrm{kD}$ protein detected by immunoblotting in four anti-OJ sera. This protein did not appear to be part of the synthetase complex, but surprisingly, affinity-purified anti-48 kD from JS serum immunoprecipitated the synthetase complex, while anti-48 $\mathrm{kD}$ from other sera with the autoantibody did not. This may indicate unique epitopes for the JS autoantibodies, also evident in the immunoblot reactivity of JS serum with IleRS.

Sera with one or more of the additional antisynthetases immunoprecipitated the same tRNA bands as those with antiIleRS alone, suggesting that the additional antisynthetases did not immunoprecipitate tRNA. While all previously recognized sera with autoantibodies against synthetases immunoprecipitated tRNAs, animal antisera against synthetases produced by immunization often do not immunoprecipitate associated tRNAs $(3,24,25)$. This suggests that reaction with particular epitopes is required for immunoprecipitation of tRNA.

It is also noteworthy that the immunoprecipitate did not appear to contain tRNAs for any other components of the complex. Most antisynthetases do not immunoprecipitate tRNA from deproteinized extract, with the exception of antiPL-12 sera (6). It was previously shown that the anti-OJ sera $\mathrm{OJ}$ and $\mathrm{NJ}$ similarly do not immunoprecipitate deproteinized 
tRNA (5). Most antisynthetases are felt to immunoprecipitate tRNA from whole extract indirectly, due to the affinity of the tRNA for the synthetase. This suggests that the tRNA-binding site is spared by antisynthetases. If this were the mechanism, however, immunoprecipitation of tRNAs for all synthetase components of the complex would have been expected. The observed tRNA bands appear to be too restricted for that to be the case, and data indicated that the immunoprecipitated tRNA was predominantly tRNA ${ }^{\text {ile }}$. This implies that antisynthetase autoantibodies are involved in immunoprecipitation of tRNA, possibly by stabilizing the synthetase-tRNA complex or increasing affinity of synthetase for tRNA. Alternatively, synthetases with higher affinity for their tRNAs may be more likely to become antigenic than other synthetases. These hypotheses are consistent with hypotheses suggesting that antisynthetases are induced by complexes of synthetase with viral RNA.

While most anti-OJ sera inhibited IleRS, most did not react with IleRS by immunoblotting. The autoantibodies to LeuRS that were suggested by this study also did not react by immunoblot. The proteins were transferred, as demonstrated by protein stain of nitrocellulose and by staining of IleRS by JS serum (Fig. 5). This suggests exclusive reactivity with conformational epitopes and not linear epitopes of the antigens. Exclusive reactivity with conformational epitopes, with negative immunoblots, although unusual in general, has been seen with other myositis-specific autoantibodies, including anti-PL-7 (26), anti-Mi-2 (17), and anti-Fer (27). Although most anti-Jo-1 sera react by immunoblot (18), this is not due to reaction with short linear epitopes. Anti-Jo-1 autoantibodies did not react when tested against each of the hexapeptides that could be derived from the entire sequence of the HisRS molecule, while animal antisera reacted with many peptides throughout the protein (24).

Thus, patients may develop autoantibodies to multiple synthetases that are components of the synthetase complex, but not to synthetases outside the complex. Sera with autoantibodies to synthetases that are not in the complex never have autoantibodies to more than one synthetase. A possible explanation is that the primary anti-OJ response is directed at IleRS, and this initial response renders the entire synthetase complex immunogenic, perhaps as a result of immunization with altered complexes containing bound anti-IleRS. This is similar to the hypothesis proposed to explain a response against multiple epitopes resulting from molecular mimicry at a single epitope (28). Extension of an initial immune response against one component to other components of a macromolecular complex has been observed in serial samples from patients with antiU1RNP and anti-Sm (29), and may be the mechanism for autoantibodies to multiple components of other complexes. The formation of anti- $160 \mathrm{kD}$, anti-LeuRS, and anti-LysRS as extensions rather than primary responses may explain the inability to demonstrate immunoprecipitated tRNA, and the lack of inhibition of $160 \mathrm{kD}$. Extension may be more like a conventional immunization than is the primary antisynthetase response. The mechanism for the primary antisynthetase response is a central question.

The three highest molecular weight components appeared to be the most abundant proteins of the immunoprecipitated synthetase complex in all immunoprecipitation gels. Although this could have resulted from higher methionine content, these bands were also stronger by protein stain of immunoprecipi- tates (Fig. 5). These components ( $160 \mathrm{kD}[a]$, IleRS [b], and LeuRS $[c])$ are not known to be overrepresented in the complex (15). A possible explanation is that trimolecular complexes of proteins $a, b$, and $c$ may exist, in addition to the full multi-enzyme complexes. Autoantibodies to one component of the trimolecular complex would immunoprecipitate both it and the full complex. A partial complex might be generated in vitro during extract preparation, or could be present in vivo. In the above hypothesis, such in vivo partial complexes could also be immunogenic after a response to IleRS, consistent with our evidence of autoantibodies to each of the three components.

The data do not exclude the possibility of reaction of antiOJ sera with additional synthetase complex components. It is possible that additional autoantibodies reactive with synthetase complex components exist that neither inhibit synthetase activity nor react by immunoblot, and were not detected. There may even be a synthetase other than IleRS that is bound by all anti-OJ sera. Even if such antibodies existed, however, the anti-lleRS would still be the most likely primary response. All four other primary antisynthetase antibody responses inhibit enzymatic activity and immunoprecipitate tRNA, properties shared by anti-IleRS; if undetected autoantibodies existed, they would have neither property. Such undetected autoantibodies should still be capable of immunoprecipitating the synthetase complex, and thus we can exclude their existence in anti-OJ-negative sera.

Although anti-OJ autoantibodies are uncommon, their unique features may provide important insights into mechanisms leading to the induction and maintenance of autoantibodies generally. These findings support the hypothesis that the responses are antigen driven, and provide an example of autoantibodies to multiple components of a macromolecular complex, which may have developed after a primary response against a single component. The studies demonstrate for the first time sera reactive with multiple synthetases, but when the details of their reactivity are considered, this is consistent with previous impressions about the independence of the five individual primary antisynthetase autoantibody responses.

\section{Acknowledgments}

The authors thank Drs. Frank C. Arnett, Chester Oddis, Paul H. Plotz, Richard Leff, and Morris Reichlin for kind referral of patient serum and clinical information on their patients with anti-OJ, and Dr. Dennis Klinman and Dr. David Finbloom for useful comments after reviewing the manuscript. We also thank Giorgio Barbi and Michael Elliott for technical assistance, and Larissa Kogan, who assisted as a Sir Alexander Fleming Scholar at the Oklahoma Medical Research Foundation.

This work was supported by grants from the National Institutes of Health (AR-32214, AI-27181, and AI-21568), and by Department of Veterans Affairs medical research funds.

\section{References}

1. Bernstein, R. M., S. H. Morgan, J. Chapman, C. C. Bunn, M. B. Mathews, M. Turner-Warwick, and G. R. V. Hughes. 1984. Anti-Jo-1 antibody: a marker for myositis with interstitial lung disease. Br. Med. J. 289:151-152.

2. Bunn, C. C., R. M. Bernstein, and M. B. Mathews. 1986. Autoantibodies against alanyl-tRNA synthetase and tRNA ${ }^{\text {ala }}$ coexist and are associated with myositis. J. Exp. Med. 163:1281-1291.

3. Mathews, M. B., M. Reichlin, G. R. V. Hughes, and R. M. Bernstein. 1984. Anti-threonyl-tRNA synthetase, a second myositis-related autoantibody. J. Exp. Med. 160:420-434.

4. Targoff, I. N., F. C. Arnett, and M. Reichlin. 1988. Antibody to threonyltransfer RNA synthetase in myositis sera. Arthritis Rheum. 31:515-524. 
5. Targoff, I. N. 1990. Autoantibodies to aminoacyl-transfer RNA synthetases for isoleucine and glycine: Two additional synthetases are antigenic in myositis. J. Immunol. 144:1737-1743.

6. Targoff, I. N., and F. C. Arnett. 1990. Clinical manifestations in patients with antibody to PL-12 antigen (alanyl-tRNA synthetase). Am. J. Med. 88:241251.

7. Targoff, I. N., and M. Reichlin. 1988. Immunological aspects. In Inflammatory Diseases of Muscle. F. L. Mastaglia, editor. Blackwell Scientific Publications, Oxford, UK. 37-70.

8. Targoff, I. N. 1992. Autoantibodies in polymyositis. Rheum. Dis. Clin. North Am. 18:455-482.

9. Nishikai, M., and M. Reichlin. 1980. Heterogeneity of precipitating antibodies in polymyositis and dermatomyositis: characterization of the Jo-1 antibody system. Arthritis Rheum. 23:881-888.

10. Mathews, M. B., and R. M. Bernstein. 1983. Myositis autoantibody inhibits histidyl-tRNA synthetase: a model for autoimmunity. Nature (Lond). 304:177-179.

11. Targoff, I. N., E. P. Trieu, P. H. Plotz, and F. W. Miller. 1992. Antibodies to glycyl-transfer RNA synthetase in patients with myositis and interstitial lung disease. Arthritis Rheum. 35:821-830.

12. Love, L. A., R. L. Leff, D. D. Fraser, I. N. Targoff, M. C. Dalakas, P. H. Plotz, and F. W. Miller. 1991. A new approach to the classification of idiopathic inflammatory myopathy: myositis-specific autoantibodies define useful homogeneous patient groups. Medicine (Baltimore). 70:360-374.

13. Yoshida, S., M. Akizuki, T. Mimori, H. Yamagata, S. Inada, and $M$ Homma. 1983. The precipitating antibody to an acidic nuclear protein antigen, the Jo-1, in connective tissue diseases: a marker for a subset of polymyositis with interstitial pulmonary fibrosis. Arthritis Rheum. 26:604-611.

14. Oddis, C. V., T. A. Medsger, Jr., and L. A. Cooperstein. 1990. A subluxing arthropathy associated with the anti-Jo-1 antibody in polymyositis/dermatomyositis. Arthritis Rheum. 33:1640-1645.

15. Mirande, M. 1991. Aminoacyl-tRNA synthetase family from prokaryotes and eukaryotes: structural domains and their implications. Prog. Nucleic Acid Res. Mol. Biol. 40:95-142.

16. Dang, C. V., and C. V. Dang. 1986. Higher eukaryotic aminoacyl-tRNA synthetases in physiologic and pathologic states. Mol. Cell. Biochem. 71:107-120.

17. Targoff, I. N., and M. Reichlin. 1985. The association between Mi-2 antibodies and dermatomyositis. Arthritis Rheum. 28:796-803.
18. Targoff, I. N., and M. Reichlin. 1987. Measurement of antibody to Jo- 1 by ELISA and comparison to enzyme inhibitory activity. J. Immunol. 138:28742882.

19. Targoff, I. N., F. C. Arnett, L. Berman, C. O'Brien, and M. Reichlin. 1989. Anti-KJ: a new antibody associated with the myositis/lung syndrome that reacts with a translation-related protein. J. Clin. Invest. 84:162-172.

20. Thommes, P., R. Fett, B. Schray, N. Kunze, and R. Knippers. 1988. The core region of human glutaminyl-tRNA synthetase homologies with the Escherichia coli and yeast enzymes. Nucleic Acids Res. 16:5391-5406.

21. Schray, B., P. Thommes, and R. Knippers. 1990. Glutaminyl-tRNA synthetase as a component of the high-molecular weight complex of human aminoacyl-tRNA synthetases: an immunological study. Biochim. Biophys. Acta. 1087:226-234.

22. Cerini, C., P. Kerjan, M. Astier, D. Gratecos, M. Mirande, and M. Semeriva. 1991. A component of the multisynthetase complex is a multifunctional aminoacyl-tRNA synthetase. EMBO (Eur. Mol. Biol. Organ.) J. 10:4267-4277.

23. Bohan, A., and J. B. Peter. 1975. Polymyositis and dermatomyositis. Parts 1 and 2. N. Engl. J. Med. 292:344-347, and 403-407.

24. Miller, F. W., K. A. Waite, T. Biswas, and P. H. Plotz. 1990. The role of an autoantigen, histidyl-tRNA synthetase, in the induction and maintenance of autoimmunity. Proc. Natl. Acad. Sci. USA. 87:9933-9937.

25. Roberts, M. M., and I. N. Targoff. 1989. Comparison of species specificity of autoimmune anti-Jo-1 and induced rabbit anti-Jo-1. Arthritis Rheum. 32:S22. (Abstr.)

26. Dang, C. V., E. M. Tan, and J. A. Traugh. 1988. Myositis autoantibody reactivity and catalytic function of threonyl-tRNA synthetase. FASEB (Fed. Am Soc. Exp. Biol.) J. 2:2376-2379.

27. Targoff, I. N., and J. Hanas. 1989. The polymyositis-associated Fer antigen is elongation factor la. Arthritis Rheum. 32:S81. (Abstr.)

28. Query, C. C., and J. D. Keene. 1987. A human autoimmune protein associated with UI RNA contains a region of homology that is cross-reactive with retroviral p30gag antigen. Cell. 51:211-220.

29. Ter Borg, E. J., G. Horst, E. Hummel, D. Jaarsma, P. C. Limburg, and C. G. M. Kallenberg. 1988. Sequential development of antibodies to specific Sm polypeptides in a patient with systemic lupus erythematosus: evidence for independent regulation of anti-double-stranded DNA and anti-Sm antibody production. Arthritis Rheum. 31:1563-1567. 